\title{
Cutaneous Leiomyosarcoma of the Face
}

\author{
Nam Gyun Kim¹, \\ Jun Oh Kim', \\ Young Ji Park', \\ Jun Sik Kim', \\ Yoon Jung Lee ${ }^{2}$, \\ Kyung Suk Lee ${ }^{1}$
}

${ }^{1}$ Department of Plastic and Reconstructive Surgery, Institute of Health Sciences, College of Medicine and Hospital, Gyeongsang $\mathrm{Na}$ tional University, Jinju;

${ }^{2}$ Department of Plastic and Reconstructive Surgery, Gyeongsang National University Changwon Hospital, Changwon, Korea

No potential conflict of interest relevant to this article was reported.
Cutaneous leiomyosarcoma is an uncommon superficial soft tissue sarcoma and mainly found in the middle aged to elderly males. It can occur in any part of the body, mostly affecting the extremities and rarely affecting the face. It grows relatively slowly, can be diagnosed by biopsy and is treated by surgical excision. It needs to be distinguished from other spindle cell neoplasms, and immunohistochemical markers are usually required to attain an accurate diagnosis. We report a case of cutaneous leiomyosarcoma appeared on the left cheek within 6 month of a 73-year-old female patient suspected with malignant melanoma before surgery

Keywords: Leiomyosarcoma / Face

\section{INTRODUCTION}

Cutaneous leiomyosarcoma is an uncommon malignant smooth muscle tumor and it occupies approximately less than 3\% of cutaneous soft tissue neoplasms with a predilection for middle-aged to elderly male. This tumor can originate either in the hair erector muscle and be located in the dermis [1]. Moreover, cutaneous leiomyosarcoma appearing in dermis and subcutaneous tissues is rare and involves approximately $2 \%-3 \%$ of all incidence of soft tissue sarcoma [2]. Morphologic differential diagnosis includes a host of other malignant spindle cell neoplasms, thereby necessitating the use of a panel of immunohistochemical markers to arrive at a definitive diagnosis [3]. Local recurrence rates after surgical excision have ranged from $14 \%$ to $42 \%$ [4]. It can be clearly diagnosed by its pathological findings. Leiomyosarcoma can occur in all parts of the body; it is predominantly found from lower

Correspondence: Kyung Suk Lee

Department of Plastic and Reconstructive Surgery, Gyeongsang National University Hospital, 79 Gangnam-ro, Jinju 52727, Korea

E-mail: opensound@hanmail.net

Received February 13, 2017 / Revised June 2, 2017 / Accepted June 3, 2017 extremities and is characterized by its comparative slow growing. In particular, leiomyosarcoma of the face is exceedingly rare, which takes up to approximately $1 \%-5 \%$ of all cases.

Cutaneous leiomyosarcoma may recur but rarely metastasize to other organisms. It has been proposed that, due to its scant metastatic potential [1]. This paper presents a rare case of leiomyosarcoma that was diagnosed as malignant melanoma on incisional biopsy, and was finally diagnosed as cutaneous leiomyosarcoma in permanent biopsy.

\section{CASE REPORT}

A 73-year-old female patient was admitted to the hospital with a wound on her left cheek after being stabbed by broken branch of a tree 6 months ago. After the incident, the patient was treated in local hospital but there was no improvement. The lesion was enlarged to the size of approximately $2 \times 3 \mathrm{~cm}$, ulcerative and firmly bulges protrude but with no tangible cervical lymph nodes to be detected (Fig. 1). With the incisional biopsy over the wound with vimentin and S-100 positive findings, it was diagnosed as suspi- 
cious of desmoplastic malignant melanoma with the negative findings of cytokeratin, CD31, CD34, HMB45, and Melan A. Positron emission tomography-computed tomography showed a hypermetabolic lesion on the left cheek area (standardized uptake value max value 14.6) (Fig. 2). However, no enhanced contrast of other lymph nodes was found.

Under general anesthesia, total excision of the left cheek lesion was carried out with safety margin of $1.5 \mathrm{~cm}$, preserving as much of the pretarsal orbicularis oculi muscle as possible. The frozen biopsy of the lesion showed tumor cell negative findings on all resected margins and base. Reconstruction of infraorbital defect was conducted with cheek rotation flap and full thickness skin graft in area that was not covered.

The post-surgical histopathologic examination of immunohistochemical staining rendered the positive findings of vimentin, smooth muscle actin, and CD34 and negative findings of cytokeratin, S-100, factor 8, HMB56, and Melan A. Thereby it was diagnosed as the cutaneous leiomyosarcoma. Immunohistochemical staining showed a positive reaction of vimentin and smooth muscle actin and in the high power field, many mitotic figures were found in 28/10 (Figs. 3, 4). No local invasion of the tumor was found from the deep and horizontal resection margins. The patient showed no post-surgical recurrence or metastasis of the tumor for about 6 months from the surgery (Figs. 5, 6).

\section{DISCUSSION}

Cutaneous leiomyosarcoma is a rare type of tumor that occurs in the skin and subcutaneous tissues, which present less than 3\% of skin and soft tissue neoplasms. Leiomyosarcoma of the skin originates from the smooth muscle around sweat glands, the arrector pili muscle of cilia and the vascular smooth muscle [4]. It occurs mainly on legs, and pre-existing leiomyomas, trauma, radioactive radiation, and chemical or ultraviolet exposures have been known as the major causes [5]. In our case, there has been a history of trauma caused from a broken branch of a tree, approximately 6 months ago.

Leiomyosarcoma of the face is extremely rare, which takes up to approximately $1 \%-5 \%$ of all soft tissue sarcomas [2]. The aver- age age of patients found with leiomyosarcoma most frequently has been known as spanning over the range of 50- to 70-years $[3,6]$. According to a prior one study, the average interval from its onset to its final diagnosis was reported to be approximately 2 years [7]. However, in our case, the cutaneous subcutaneous leiomyosarcoma was found in the patient's face and the interval from

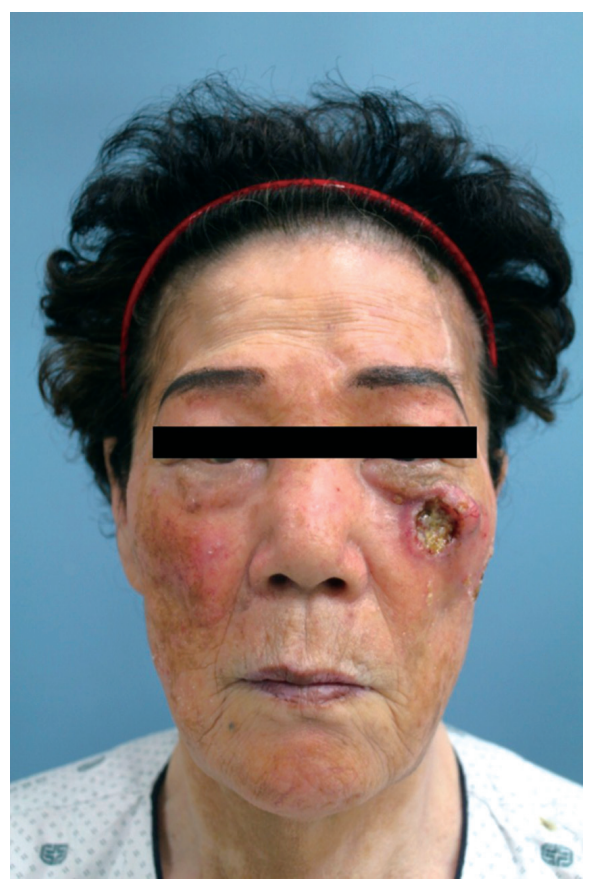

Fig. 1. A 73-year-old female presented with $2 \times 3 \mathrm{~cm}$ ulcerative wound on her left cheek.

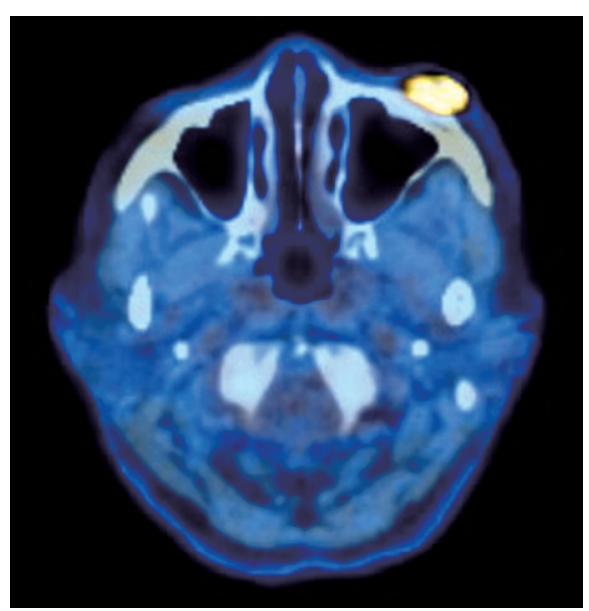

Fig. 2. Positron emission tomography-computed tomography showed hypermetabolic lesion on the left cheek area (standardized uptake value max value 14.6). 


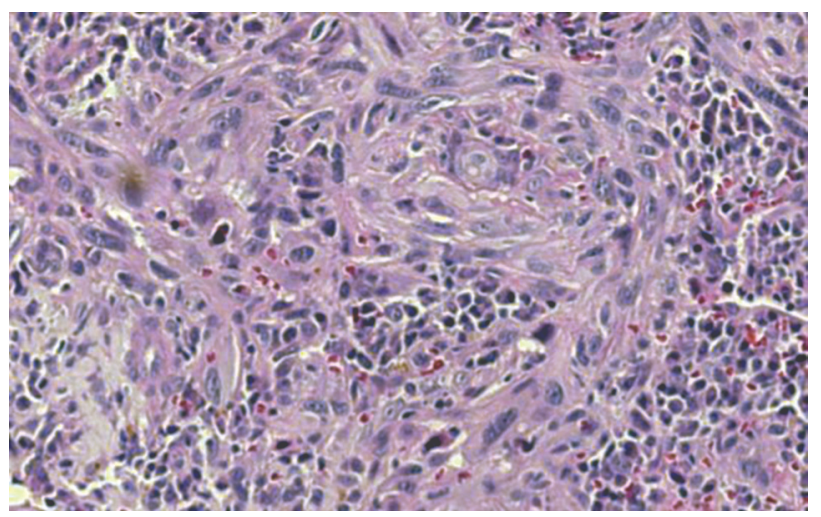

Fig. 3. Histopathologic findings of the lesion revealed composed of spindle cell with scattered pleomorphic nuclei with mitosis and hyperchromatic nuclei $(\mathrm{H} \& \mathrm{E}, \times 100)$.

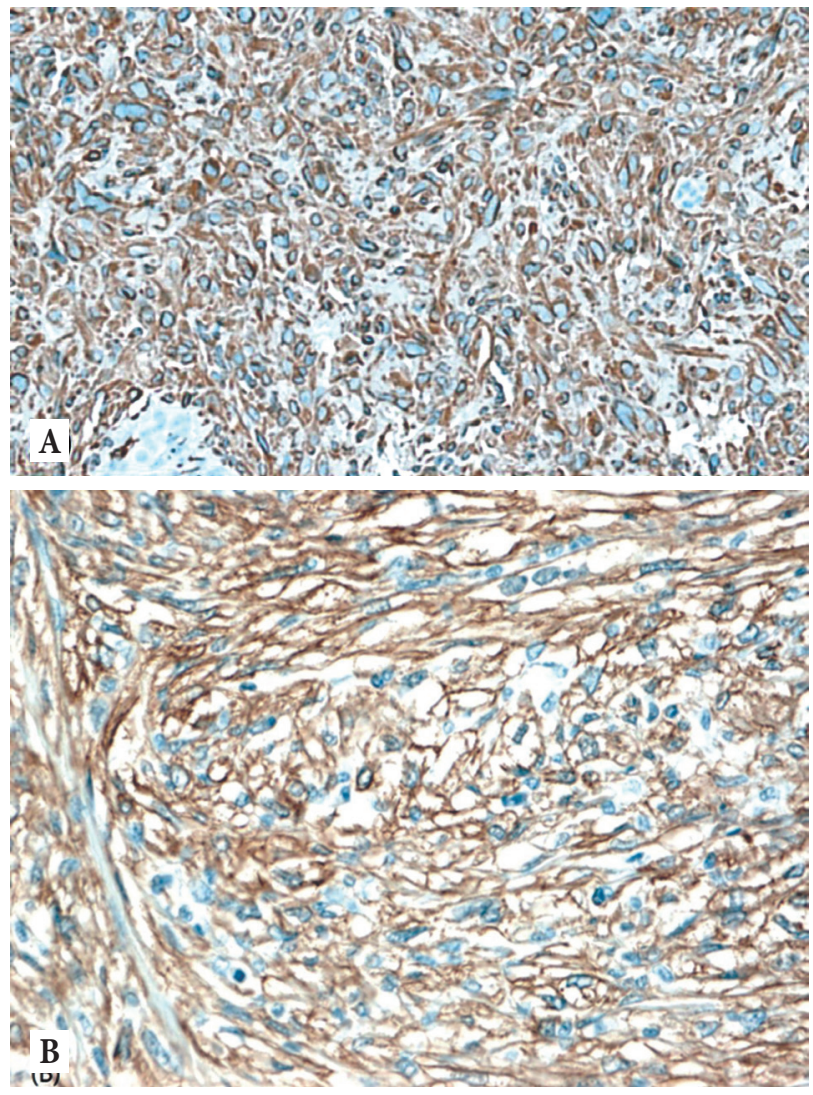

Fig. 4. Results of immunohistochemical staining showed a positive reaction of vimentin (x100) (A) and smooth muscle actin $(\times 400)(B)$.

its onset by trauma to its diagnosis was 6 months.

Leiomyosarcoma should be distinguished from atypical fibroxanthoma, dermatofibrosarcoma protuberans, angiosarcoma, and spindle cell squamous cell carcinoma through histological

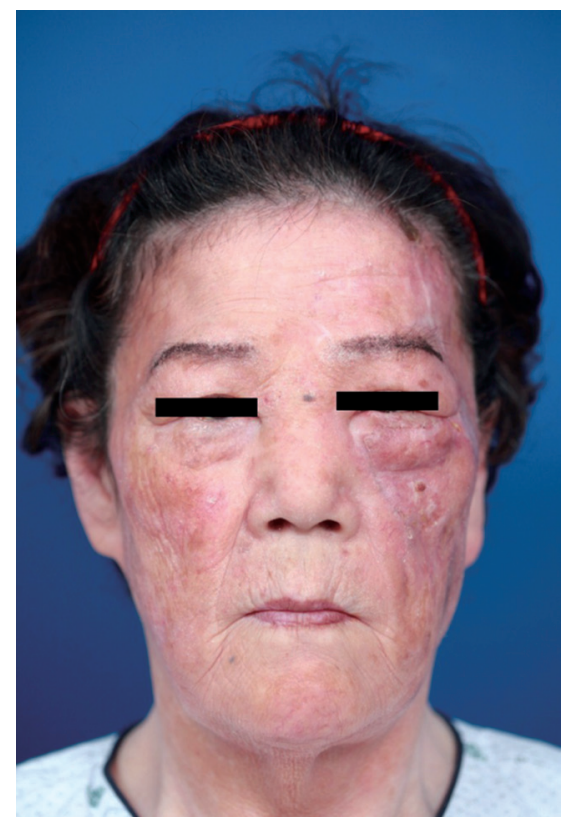

Fig. 5. The patient shows no post-surgical recurrence or metastasis of the tumor for about 6 months after surgery.

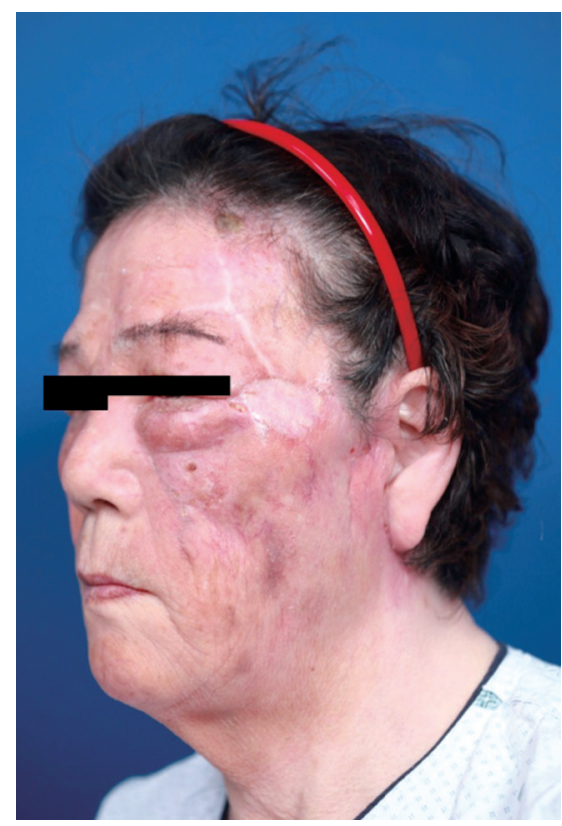

Fig. 6. The patient shows no post-surgical recurrence or metastasis of the tumor for about 6 months from the surgery.

examinations [8]. In cutaneous leiomyosarcoma, positive findings are shown in vimentin, desmin, and smooth muscle actin staining. Smooth muscle actin is more sensitive than vimentin and desmin, but it is not highly specific [6]. CK, S-100, and CD34 are 
rarely found as positive, but mostly with negative results. It is difficult to distinguish from some other tumors and if S-100 is found positive, then it should be discriminated from different skin cancer. It is known that desmoplastic malignant melanoma should be discriminated by CK and HMB45 staining [9]. In particular, the CK and S-100 immunity are known as useful immunoassays to differentiate it from other spindle cell neoplasms, squamous cell carcinomas, and malignant melanoma [10]. In our case, from the post-surgical immunochemical examination taken of all tissues, the vimentin, smooth muscle actin, and CD34 were found positive and the CK and S-100 were found negative thereby the lesion was finally diagnosed as leiomyosarcoma. Therefore, careful examination of cytological details in multiple sections, clinicopathological correlation and immunohistochemistry are necessary for definitive diagnosis.

According to Jensen et al. [11], tumor size of $\geq 5 \mathrm{~cm}$, deep location with fascia involvement, and high malignancy grade IIIB are all poor prognostic factors. The 5-year survival rate of this group is estimated to be $30 \%-40 \%$. The tumors were classified with regard to tumor grade I (low grade, 3\%), II (intermediate, 12\%), IIIA (high grade, 46\%) and IIIB (high grade, 39\%). The patient in our case, it corresponds to grade IIIB.

The most effective treatment for cutaneous leiomyosarcoma would be a wide resection that includes approximately $3-5 \mathrm{~cm}$ of normal tissue to prevent recurrence, and should be resected to the depth of subcutaneous tissue and fascia $[2,3,12]$. In our case, because it was diagnosed as a malignant melanoma in the frozen section during surgery, we excised the lesion with safety margin of $1.5 \mathrm{~cm}$. Therefore, we need to monitor the patient due to possibility of a recurrence of leiomyosarcoma for a long time. The local recurrence rate of cutaneous leiomyosarcoma is known to range from $30 \%$ to $50 \%$. Therefore a long-term follow-up is necessary regarding the possibility of distant metastasis that may involve lung, liver, or bone [3].

We have reported the case of leiomyosarcoma that was initially thought to be a malignant melanoma. Different from other cases of leiomyosarcomas, it has occurred on the patient's face, had grown rapidly over a relatively short period of 6 months after trauma, with high malignancy grade (IIIB). It can be considered that leiomyosarcoma requires early diagnosis and wide surgical excision due to the low survival rate compared to other skin cancers. After 6 months, recurrence of the cancer was not seen and mild ectropion was observed.

\section{REFERENCES}

1. Aneiros-Fernandez J, Antonio Retamero J, Husein-Elahmed H, Ovalle F, Aneiros-Cachaza J. Primary cutaneous and subcutaneous leiomyosarcomas: evolution and prognostic factors. Eur J Dermatol 2016;26:9-12.

2. Lin JY, Tsai RY. Subcutaneous leiomyosarcoma on the face. Dermatol Surg 1999;25:489-91.

3. Bali A, Kangle R, Roy M, Hungund B. Primary cutaneous leiomyosarcoma: a rare malignant neoplasm. Indian Dermatol Online J 2013;4:188-90.

4. Porter CJ, Januszkiewicz JS. Cutaneous leiomyosarcoma. Plast Reconstr Surg 2002;109:964-7.

5. Lee KC, Kim MS, Choi H, Na CH, Shin BS. Rapid growing superficial cutaneous leiomyosarcoma of the face. Ann Dermatol 2013;25:237-41.

6. Limaiem F, Chelly I, Bellil S, Mekni A, Nidhameddine K, Haouet S, et al. Primary cutaneous leiomyosarcoma: a histological and immunohistochemical study of 4 cases. Pathologica 2007;99:415-9.

7. Fields JP, Helwig EB. Leiomyosarcoma of the skin and subcutaneous tissue. Cancer 1981;47:156-69.

8. Blaise G, Nikkels AF, Quatresooz P, Hermanns-Le T, Pierard GE. Childhood cutaneous leiomyosarcoma. Pediatr Dermatol 2009;26: 477-9.

9. Auroy S, Contesso G, Spatz A, Genin J, Margulis A, Lecesne A, et al. Primary cutaneous leiomyosarcoma: 32 cases. Ann Dermatol Venereol 1999;126:235-42.

10. Annest NM, Grekin SJ, Stone MS, Messingham MJ. Cutaneous leiomyosarcoma: a tumor of the head and neck. Dermatol Surg 2007;33: 628-33.

11. Jensen ML, Jensen OM, Michalski W, Nielsen OS, Keller J. Intradermal and subcutaneous leiomyosarcoma: a clinicopathological and immunohistochemical study of 41 cases. J Cutan Pathol 1996;23:45863.

12. Fish FS. Soft tissue sarcomas in dermatology. Dermatol Surg 1996;22:268-73. 